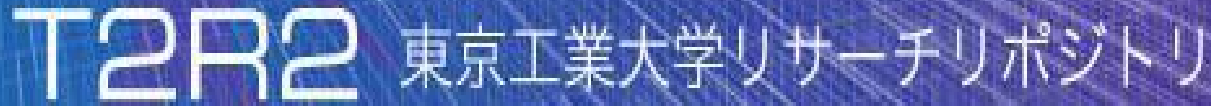

\section{Tokyo Tech Research Repository}

\section{論文 /著書情報 \\ Article /Book Information}

\begin{tabular}{|l|l|}
\hline Title & Study on Roller-Walker - Energy Efficiency of Roller-Walk - \\
\hline Author & Gen Endo, Shigeo Hirose \\
\hline Issue date &,
\end{tabular}




\title{
Study on Roller-Walker - Energy Efficiency of Roller-Walk -
}

\author{
Gen Endo and Shigeo Hirose
}

\begin{abstract}
Roller-Walker is a leg-wheel hybrid mobile robot using a passive wheel equipped on the tip of each leg. The passive wheel can be transformed into sole mode by rotating ankle roll joint when Roller-Walker walks on rough terrain. This paper discuss energy efficiency of locomotion in wheeled mode. We define a leg trajectory to produce forward straight propulsion and discuss the relationships between the parameters of the leg trajectory and energy efficiency of the propulsion using numerical simulator. We find optimum parameter sets where optimization criteria is specific resistance. Then we carried out hardware experiments and empirically derived experimental specific resistance. We show that wheeled locomotion has 8 times higher energy efficiency than ordinary crawl gait. Finally we compare the specific resistance of Roller-Walker with other walking robots described in the literatures.
\end{abstract}

\section{INTRODUCTION}

A walking robot which can select a discrete foot placement with an articulated leg has potential capabilities: 1) it can move adaptively on rugged terrain, 2) it has higher energy efficiency than a wheeled vehicle on soft deformable terrain because it leaves discrete footprints whereas a wheeled vehicle makes continuous furrow which requires larger traction force, 3) it makes holonomic and omnidirectional motion without slip, 4) it can be a stable and movable platform for a manipulator even on rugged terrain when it is not walking. Many walking robots have been developed to move on rugged terrain so far and nowadays some robots edge closer to practical use [1][2].

However, on hard flat terrain, wheeled locomotion is absolutely better than legged locomotion in terms of moving velocity and energy efficiency. Therefore, many research attempt to combine the advantages of these two types of locomotion through leg-wheel hybrid vehicles [3][4][5][6].

In these previous studies, most of the hybrid vehicles equipped with driven wheels, which requires actuators to drive the wheels. Since driven wheels tend to be heavy and bulky, the hybrid vehicles increased the total weight of the robot. We consider that increasing the weight of the robot due to hybridization has a serious defect in walking performance because the walking vehicle is already heavy enough due to many degrees of freedom in the leg mechanisms.

Therefore we have proposed a leg-wheel hybrid vehicle with passive wheels, which are the simplest and lightest wheels [7]. Passive wheels can minimize additional weight for hybridization and do not reduce potential walking performance of the walking robot. Fig. 1 shows overview of

Gen Endo and Shigeo Hirose are with Department of Mechanical and Aerospace Engineering, Tokyo Institute of Technology, \#I1-60, 2-12-1, Ookayama, Meguro-ku, Tokyo, 152-8552, Japan

\{gendo, hirose\}@mes.titech.ac.jp
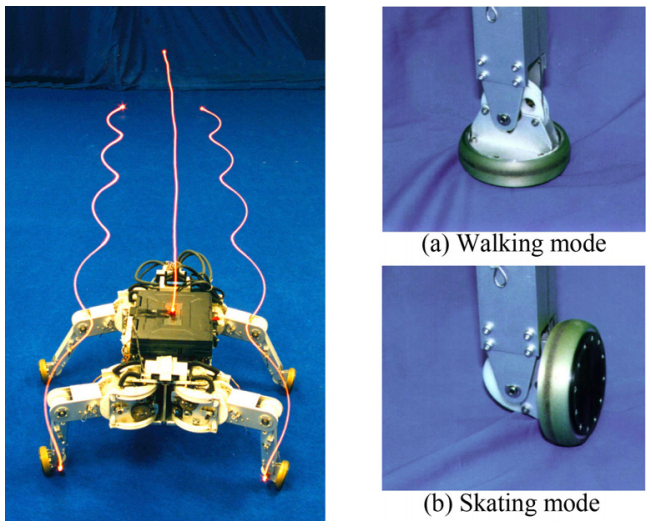

Fig. 1. Roller-Walker: the white lines show trajectories of the frontal leg ends and the body.(left), passive wheel in two modes(right))

the prototype robot of Roller-Walker. Roller-Walker equips with a passive wheel on the tip of each leg, and the passive wheel can be transformed into sole mode by rotating the ankle roll joint when Roller-Walker walks on rough terrain. Roller-Walker can propel efficiently by means of the same principle of roller-skating on the flat ground. Roller-Walker has distinctive advantages as follows:

1) Minimizing additional weight

2) Applicable to the previous walking robots

3) High power propulsion using legs' actuators

4) Potential capability of the terrain adaptation

In our previous work, we named the wheeled locomotion as "Roller-Walk" and derived basic leg trajectories such as straight, circular and rotational propulsion, where the evaluation criteria is the maximization of velocity. We verified the achievements of propulsion both in numerical simulations and hardware experiments [7]. We also proposed the leg trajectory adjustment method to track specified velocity on the friction changing ground, and discuss the relationships between the leg trajectory and propulsive force / velocity characteristics in detail [8].

However, an improvement of locomotion efficiency by Roller-Walk remains a qualitative consideration and we have not discussed yet. To the best of our knowledge, there is no detailed report addressing quantitative effectiveness of passive wheel locomotion in terms of energy efficiency in the previous studies including biped robots with passive wheels [9][10].

In this paper, we focus on the relation between the leg trajectory of Roller-Walk and energy efficiency of locomotion. We choose specific resistance [11] as an evaluation criteria and optimize the leg trajectory parameters by using a numer- 
ical simulator. Then we carried out hardware experiments to measure electrical power consumption and confirmed that the results of hardware experiments had similar tendency of the numerical simulations. Finally, we compare efficiency of Roller-Walk with that of crawl gait, which is typical walking of a quadruped. We also show the comparison of Roller-Walker's specific resistance with other walking robots described in the literatures.

\section{Simulation Model}

In this paper, we choose specific resistance at steady state as an evaluation criteria, which can be calculated by power consumption and velocity during locomotion as we are to mention in the next section. To derive specific resistance, we develop a simplified numerical simulator. In this section, we explain a kinematic model and method of numerical analysis.

There is an infinite of possibilities for the leg trajectory within leg's workspace to produce propulsive motion in wheeled mode. To simplify the problem, we assume that; 1) all legs are in support phase, 2) all legs are massless and center of gravity of the robot is located at the middle of the body, 3) left-and-right legs move symmetric and periodic. Fig. 2 shows a coordinate system for a numerical analysis. The axis of the passive wheel is fixed to the leg at a right angle and its camber angle is also kept at a right angle. We assume a symmetric leg trajectory as follows:

$$
\begin{aligned}
& d(t)=d_{\text {offset }}+d_{0}(\sin (\omega t+3 \pi / 2)+1), \\
& \theta(t)=-\theta_{0} \sin (\omega t+3 \pi / 2+\phi) .
\end{aligned}
$$

$d_{0}$ and $\theta_{0}$ are amplitudes of sinusoidal oscillation in the normal and tangential directions of the passive wheel, respectively. $\omega$ determines an angular velocity of the oscillation. $\phi$ is a phase difference between the oscillations in the normal and tangential direction. (Here, we introduce appropriate offsets considering initial posture and leg's workspace of the hardware prototype.) There are four control parameters, $d_{0}, \theta_{0}, \omega, \phi$, to modulate the leg trajectory in Eqn.(1),(2). An example of the leg trajectory is illustrated in Fig. 2. We assume Coulomb friction at a contact point of the passive wheel on the ground, and thus the resulting tangential force $F_{t}(t)$ and normal force $F_{n}(t)$ due to the periodic leg motion can be expressed as follows:

$$
\begin{aligned}
& F_{t}(t)=-\operatorname{sgn}(V \cos \theta(t)+d(t) \dot{\theta}(t)) \cdot \mu_{t} \cdot m g / 4 \\
& F_{n}(t)=-\operatorname{sgn}(V \sin \theta(t)+\dot{d}(t)) \cdot \mu_{n} \cdot m g / 4
\end{aligned}
$$

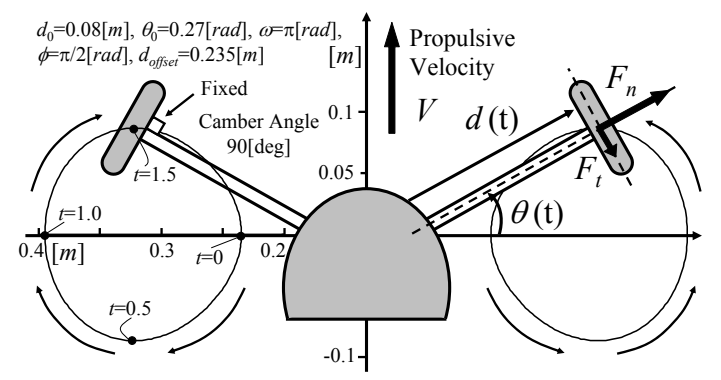

Fig. 2. Simulation model and an example of the leg trajectory(left)
Here, $\operatorname{sgn}(*)$ is signum function and $V$ is propulsive velocity. $\mu_{t}, \mu_{n}$ are Coulomb friction coefficients in the tangential and normal direction, respectively. $m$ is a total mass of the robot and $g$ is gravity. The same kinematic model is applied to the hinder legs and we introduce a phase difference of $\phi_{f r}=3 \pi / 2 \mathrm{rad}$ between the frontal and hinder legs in order to minimize velocity fluctuation at steady state [7]. Since leg motions are symmetric, the lateral forces are canceled each other out and the sagittal forces remain as a traction force. We obtain activated acceleration of the robot by using the traction force divided by $m$. Finally, we calculate propulsive velocity $V$ by numerical integration of the acceleration. In our study, numerical simulation time step is set to $10 \mathrm{~ms}$.

Additionally, we can derive a necessary condition of propulsion as follows:

$$
\left|F_{n}(t) \sin \theta(t)\right|>\left|F_{t}(t) \cos \theta(t)\right| .
$$

Here, by substituting Eqn.(3)(4) in above equation, we obtain

$$
|\theta(t)|>\tan ^{-1}\left(\mu_{t} / \mu_{n}\right)
$$

Eqn.(6) indicates that $\theta(t)$ to perform propulsion should be larger than the minimum value which is determined by a ratio of friction coefficients of the tangential and normal direction.

We show an example of the result of velocity simulation with a hardware experiment in Fig. 3. Simulated velocity is close to the measured actual velocity with an accuracy of $10 \%$ error when it reaches steady velocity, suggesting that the velocity simulation has sufficient accuracy for the following parametric study.

\section{SPECIFIC RESISTANCE}

A dimensionless quantitiy specific resistance $\varepsilon$ [11] is widely used to evaluate efficiency of locomotion.

$$
\varepsilon=\frac{E}{W \cdot L}
$$

Here, $E$ is required energy for locomotion and $W$ is weight of the vehicle and $L$ is a traveling distance. We can rewrite the above equation with power consumption $P$ and velocity $V$ by differentiating both numerator and denominator as follows.

$$
\varepsilon=\frac{d E / d t}{W \cdot d L / d t}=\frac{P}{W \cdot V}
$$

The smaller specific resistance indicates the higher energy efficiency. For examples, efficiency of a sliding object whose

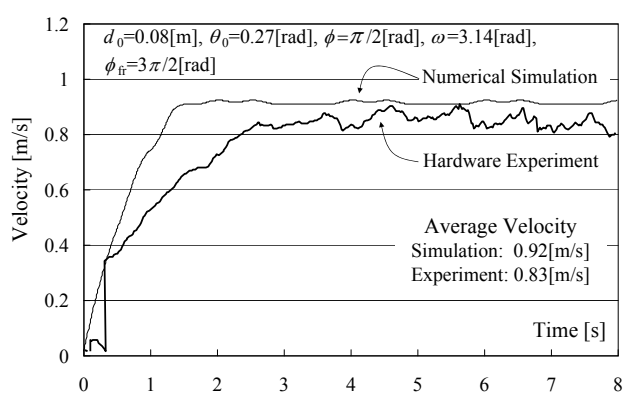

Fig. 3. An example of a numerical simulation 


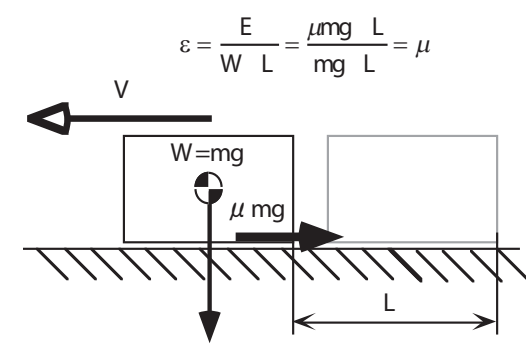

Fig. 4. Examples of specific resistance: work against friction resistance(left), work against gravity(right)

dynamic friction coefficient equals to $\mu$ is shown in Fig. 4 (left) as well as the case of vertical lifting against the gravitational force (right). In the case of the sliding object, $\varepsilon=\mu$ and the smaller $\mu$ provides the higher efficiency of locomotion, which agrees with intuitive understanding. In the case of object lifting, $\varepsilon$ becomes 1 .

\section{Numerical Simulation}

In this section, we derive an optimum leg trajectory for a straight steady propulsion which minimizes specific resistance. It is difficult to estimate general power consumption because total power consumption always depends on various factors such as mechanical power output, hardware configuration, efficiency of actuator system, efficiency of power source and so on. In this section, we focus on a relation between the leg trajectory and pure mechanical power consumed by passive wheels to eliminate the hardware specific problems. We calculated power consumption from the product of the resistant force times moving velocity of the passive wheel.

Here we have four control parameters, $\left(d_{0}, \theta_{0}, \omega, \phi\right)$, to modulate the leg trajectory. In our previous work, we studied the optimum parameters to achieve maximum velocity [7] and found that the generated velocity $V$ is proportional to $\omega$ and $\phi=\pi / 2$ is desirable. As for $\omega$, the result is reasonable because Eqn.(3)(4) do not depend on the magnitudes of $V$ and thus the larger $\omega$ supplies the larger propulsive power proportional to $\omega$. Therefore $\omega$ does not affect to specific resistance in Eqn.(8). As for $\phi$, we can analytically derive a geometrical constraint $\phi=\pi / 2$, where the passive wheel does not generate velocity in the normal direction assuming that $V$ is constant and $\theta(t)$ is described by a sin-wave. Therefore we investigate $\left(d_{0}, \theta_{0}\right)$ in detail.

Fig. 5 shows simulated specific resistance where $\mu_{t}=$ $0.026, \mu_{n}=0.417$ obtained by a preliminary experiment using a hardware passive wheel on vinyl floor sheet. As for $d_{0}$, the larger $d_{0}$ makes the lower specific resistance. However $d_{0}$ has a hardware limitation of leg's workspace whose maximum value is $0.08 m$ in case of our experimental hardware. As for $\theta_{0}$, the optimum value is $0.17 \mathrm{rad}$ which is slightly larger than that of the maximum velocity $(0.15 \mathrm{rad})$ [7]. Specific resistance rapidly increases when $\theta_{0}$ is smaller than 0.1 because it violates the sufficient condition in Eqn.(5).

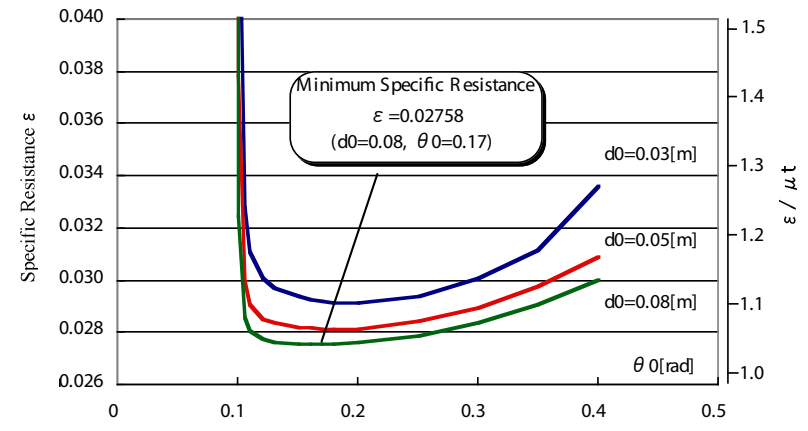

Fig. 5. Relationship between the leg trajectory parameters $\left(d_{0}, \theta_{0}\right)$ and simulated specific resistance

The vertical axis on the right in Fig. 5 indicates the ratio of specific resistance $\varepsilon$ and the tangential friction coefficient $\mu_{t}$. The ratio $\varepsilon / \mu_{t}$ becomes 1.06 at the minimum specific resistance, suggesting that the optimized leg trajectory hardly generates a slipping loss in the normal direction and required power is almost all consumed by a rolling resistance in the tangential direction. Therefore we can consider the optimized trajectory described by simple sinusoidal equations (Eqn.(1)(2)) are sufficient to generate energy efficient wheeled locomotion.

\section{HARDWARE EXPERIMENT}

In the previous section, required power with the optimized trajectory is almost the same power for the rolling resistance of passive wheels. However it is difficult to accurately measure the tangential friction because the absolute value is very small. Moreover, accurate mechanical power measurement without suffering from the mechanical loss of the driving system is extremely difficult. On the other hand, a total electric power measurement is very easy and beneficial to improve the actual hardware system. Therefore we obtain experimental specific resistance by measuring electric power, which is the product of current and voltage supplied by the installed battery. Of course electric power consumption includes various losses as we mentioned before and we can not directly compared the absolute value of simulated specific resistance with experimental specific resistance. However we can still discuss the relation between the leg trajectory and specific resistance and compare the tendency of hardware specific resistance with respect to the idealized mechanical specific resistance.

\section{A. Measurement Method}

We measured battery current by using a current sensor (NEC/TOKIN MDCS) and also measured battery voltage. At the beginning of the experiment, we measured power consumption as "basal metabolism" where Roller-Walker took the standard posture and the main body was supported by a stand in order to lift up all legs from the ground. To derive net specific resistance, we subtracted this basal metabolic power from measured total power of locomotion experiments. We confirmed that basal metabolism power 
is $82.0 \mathrm{~W}$, which is mainly consumed by 12 servo driver circuits.

We also measured the velocity using a tachogenerator attached to an additional passive wheel for dead reckoning under the main body. Fig. 6 shows a snapshot of the measurement experiment on the vinyl floor. All measurement devices and a control computer were mounted on the main body and the total weight of the robot was $37.6 \mathrm{~kg}$. We set a low body height in order to maximize $d_{0}$. We manually adjusted the leg parameters as well as a $\theta(t)$ offset added for steering motion to control velocity and direction until steady straight propulsion was established, which typically took $50 \mathrm{~m}$ travelling distance. We did twice measurements for each parameter set and averaged the results.

\section{B. Specific resistance in Roller-Walk}

To verify the relation between leg trajectory parameters and specific resistance, we did parametric measurement. Firstly, we discuss $\omega$ which determines the frequency of the leg trajectory. As we expected, Fig. 7 indicates that the larger $\omega$ produces larger velocity and power consumption although net specific resistance keeps relatively constant. Thus this result supports our physical understanding mentioned in Section IV, that is $\omega$ does not affect to specific resistanse. Moreover this result also suggests that a propulsive velocity control adjusting $\omega$ can be achieved without affecting efficiency of locomotion. This is a nice and useful characteristic because once we find energy efficient optimum leg trajectory, we can keep the maximum efficiency regardless of the propulsive velocity.

Secondly, we discuss $d_{0}$, the amplitude of the nominal oscillation, which also relates to the propulsive energy input. Fig. 8 shows that the larger $d_{0}$ achieves larger velocity and power, and specific resistance slightly decreases with the larger $d_{0}$. Thus we define the maximum $d_{0}=0.08 \mathrm{~m}$ within leg's workspace as the optimum value.

Thirdly, we discuss $\theta_{0}$, the amplitude of the tangential oscillation, which can be considered as a reduction ratio of the propulsive movement [8]. In Fig. 9, we could not carry out the measurement where $\theta_{0}$ is less than $0.13 \mathrm{rad}$ because Roller-Walker could not propel and required joint torques hit current limitations of electric system. (Thus, it is expected that hardware specific resistance would rapidly increase where $\theta_{0}$ is less than 0.13.) Although measured specific resistance is about 20 times higher than the simulated result shown in Fig. 5, the tendency of the relation with $\theta_{0}$ in hardware measurement is similar to that of numerical simulation. The smaller $\theta_{0}$ decreases specific resistance and it takes minimum value at $\theta_{0}=0.15 \mathrm{rad}$ where the velocity is also maximized.

Finally, we empirically derived the optimum parameter set which minimizes specific resistance using this prototype. We obtained minimum specific resistance of 0.44 where $d_{0}=$ $0.08 \mathrm{~m}, \theta_{0}=0.15 \mathrm{rad}, \omega=6.28 \mathrm{rad} / \mathrm{s}, \phi=\pi / 2, \phi_{f r}=3 \pi / 2$. Required total power was $452 \mathrm{~W}$ and achieved velocity was $2.27 \mathrm{~m} / \mathrm{s}$, which is about 11 times faster than the walking velocity.

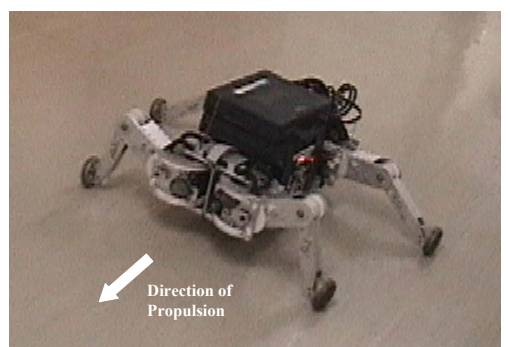

Fig. 6. Measurement of experimental specific resistance (lowering body height provides the larger $d_{0}$.)

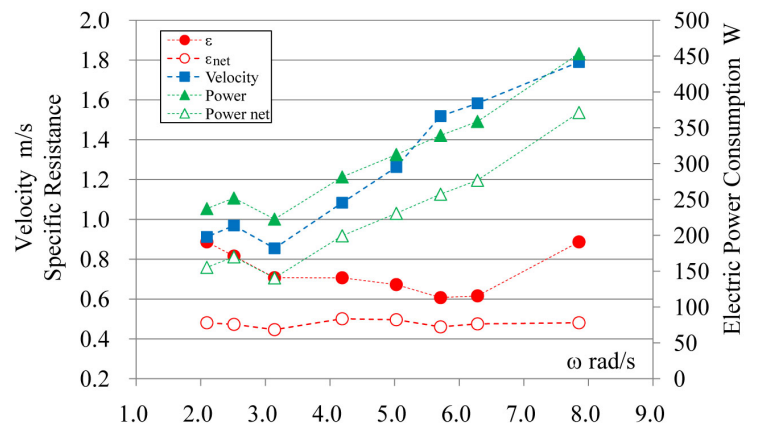

Fig. 7. Relation between $\omega$ and experimental specific resistance, velocity and power: $d_{0}=0.05 \mathrm{~m}, \theta_{0}=0.15 \mathrm{rad}, \phi=\pi / 2 \mathrm{rad}, \phi_{f r}=3 \pi / 2 \mathrm{rad}$.

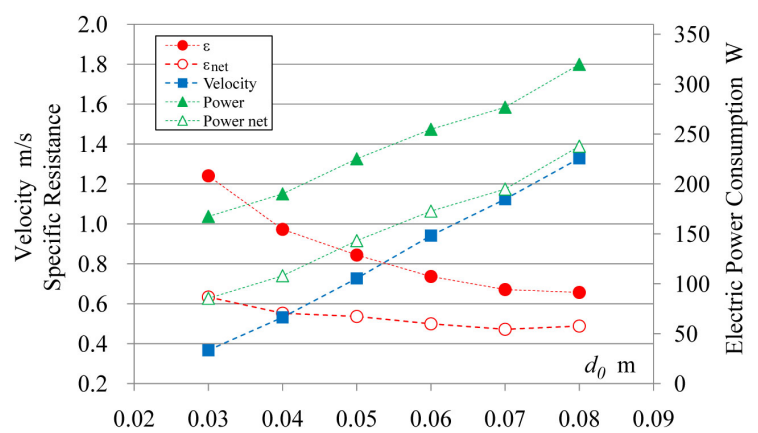

Fig. 8. Relation between $d_{0}$ and experimental specific resistance velocity and power: $\theta_{0}=0.15 \mathrm{rad}, \omega=3.14 \mathrm{rad} / \mathrm{s}, \phi=\pi / 2 \mathrm{rad}, \phi_{f r}=3 \pi / 2 \mathrm{rad}$.

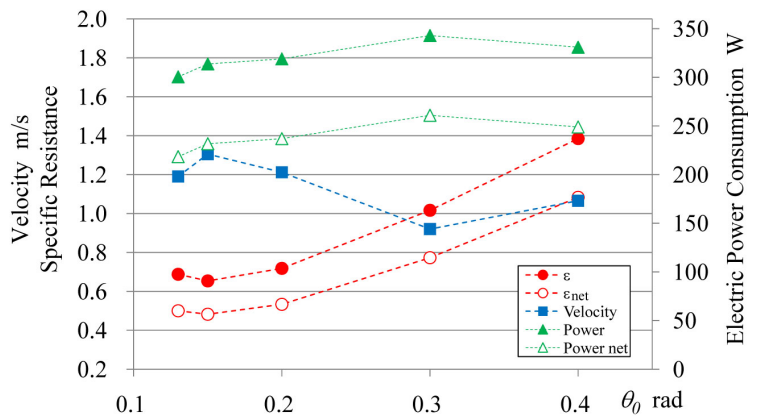

Fig. 9. Relationship between $\theta_{0}$ and experimental specific resistance velocity and power: $d_{0}=0.08 \mathrm{~m}, \omega=3.14 \mathrm{rad} / \mathrm{s}, \phi=\pi / 2 \mathrm{rad}, \phi_{f r}=3 \pi / 2 \mathrm{rad}$.

\section{Specific Resistance in Crawl Gait}

We measured specific resistance in crawl gait which is the most typical static walking of quadrupeds. The standard walking posture was selected as in [12] and the step length and height are $0.18 \mathrm{~m}$ and $0.05 \mathrm{~m}$, respectively. We used fixed duty factor of 0.75 and modulated the walking period to control the walking velocity from $0.01-0.12 \mathrm{~m} / \mathrm{s}$. When the walking velocity is larger than $0.04 \mathrm{~m} / \mathrm{s}$, we verified that net specific resistance is almost constant of 3.53 which is about 


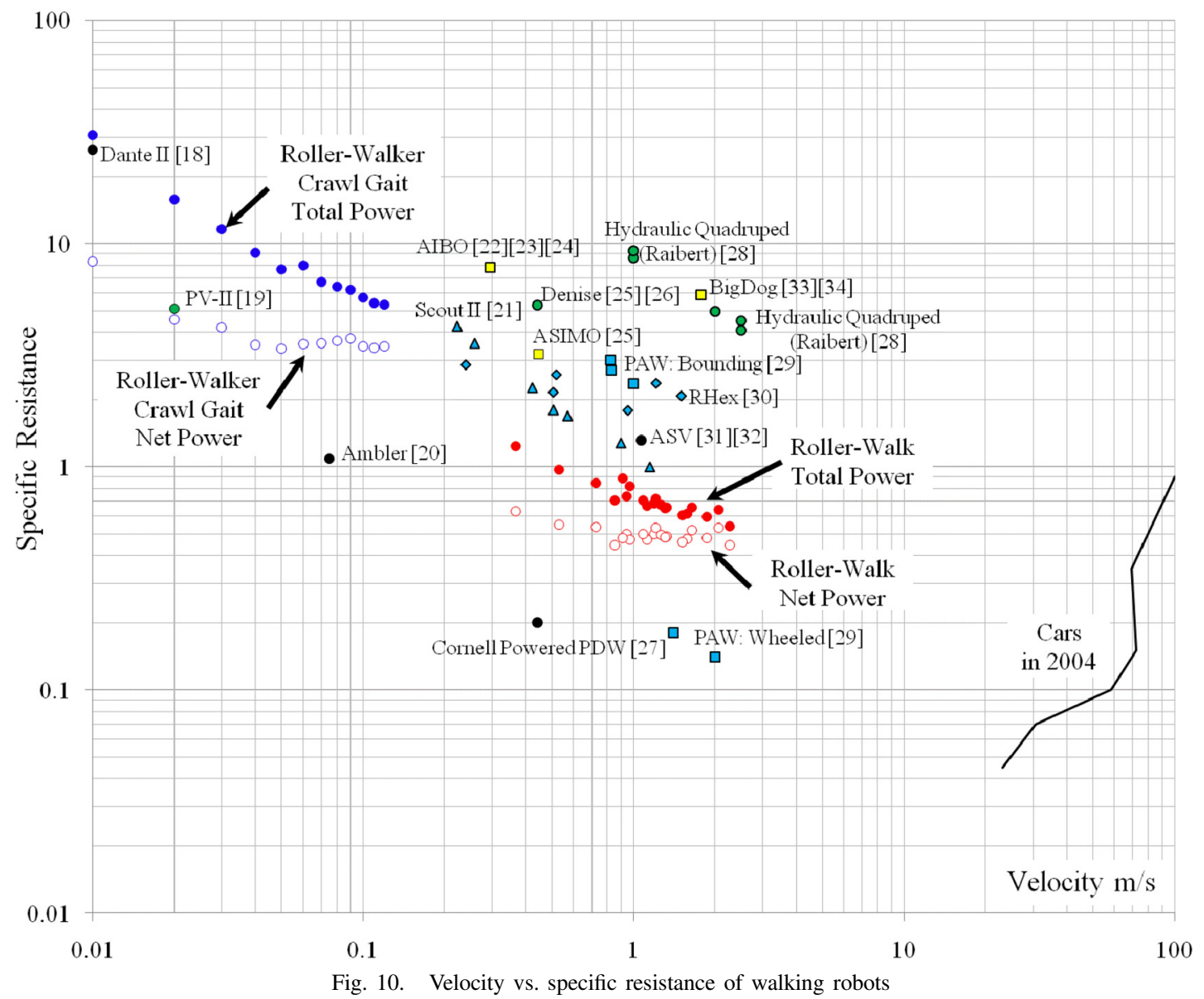

8 times higher than that of Roller-Walk. This result suggests that Roller-Walk remarkably improves energy efficiency of locomotion on the level ground. More detailed data is shown in Fig. 10.

\section{COMPARISON WITH OTHER WALKING ROBOTS}

It is very interesting and attractive to compare specific resistance of the walking robots developed so far. However, it is sometimes misleading because one robot was developed to achieve high energy efficiency on the well-prepared ground and the other robot was developed to achieve a special mission negotiating over a rough terrain. Moreover, technically speaking, the derivation of power consumption is very controversial. One robot uses pure mechanical power of an actuator shaft and the other uses total electric power supplied to the robot system which includes many devices independent of locomotion itself. Nevertheless, it seems to be still beneficial to compare specific resistance to understand basic performance of energy efficiency.

We calculated specific resistance with respect to velocity in Fig. 10. The robots in the right-bottom side possess high performance of locomotion. This diagram was originally proposed by G. Gabrielli and T.H. von Karman in [11] and several researchers updated the diagram from the viewpoint of robotics research such as [13] [14]. However several data mentioned in [13] [14] remain unclear how to derive their specific resistance and some references are very difficult to obtain at present. Therefore we dare not to directly refer to data in [13] [14] but refer to the original references listed in [13] [14]. The reference [15] also provides us with useful references to reach the original papers. Additionally, we also investigated the old but easily available online literatures such as technical reports open to the public, as well as the new walking robot in the last decade.

We focus on total power consumption derived from a power source. Thus we do not plot specific resistance derived by pure mechanical power such as ARL Monopod [13] and McGeer's Passive Dynamic Walker [16]. Green dots are calculated from measured power supplied to the actuators while black dots uses measured power supplied to the robot system. Light blue dots are also calculated from battery power to the robot system, but these robots use similar platform with a springy leg. Yellow dots are estimated from a duration of batteries or maximum power output of an equipped engine. (Detailed derivation for each specific resistance is omitted here due to a space limitation. We plan to report it as well as a comparison using pure mechanical power in the next paper.)

Fig. 10 clearly indicates that Roller-Walk improves locomotion performance compared with crawl gait. Roller-Walk is more energy efficient than any other rapid walkers with a springy leg except for PAW in wheeled mode and powered passive dynamic walker developed in Cornell university, which is a special elaborated biped to achieve extremely high energy efficiency. Moreover, there is a possibility to increase energy efficiency of the conventional robots by using Roller- 
Walker concept because it only requires a slight mechanical modification.

One might think that Roller-Walk is better than usual wheeled locomotion. As a comparison, we show a black line indicating cars in 2004 adopted from [17]. Roller-Walk does not stand a chance of competing with cars because a legged robot usually requires many degrees of freedom with a servo drive system which suffers from low efficiency. Thus, if target environment is a completely smooth terrain, conventional wheeled robot is absolutely better than RollerWalk. (This is also suggested by PAW in wheeled mode.) However we believe that Roller-Walk provides a walking robot, which is originally developed to negotiate a rugged terrain, with a practical solution to increase locomotion efficiency on the flat ground.

\section{CONCLUSION}

In this paper, we focus on the relation between the leg trajectory of Roller-Walk and energy efficiency of locomotion. We investigate specific resistance of Roller-Walk using both numerical simulations and hardware experiments. We confirmed that Roller-Walk improves locomotion efficiency 8 times higher than crawl gait. And we compare the results with other walking robots, suggesting that Roller-Walk can be an effective solution to increase efficiency of a walking robot on the level ground.

In this paper, we measured specific resistance on vinyl floor in an indoor environment. Specific resistance of RollerWalk largely depends on the frictional coefficient in the tangential direction. Thus there is a case that walking has higher energy efficiency than that of Roller-Walk when the tangential friction is very large such as walking on sand area. Automatic mode selection using energy efficiency from wheel to sole and vice versa forms one of our interesting future research topic.

\section{REFERENCES}

[1] T. Doi, R. Hodoshima, Y. Fukuda, S. Hirose, J. Mori, and T.Okamoto, "Development of a quadruped walking robot to work on steep slopes, titan XI (walking motion with compensation for compliance)," in Proc. of Int. Conf. on Intelligent Robots and Systems, 2005, pp. 3413-3418.

[2] http://www.gearlive.com/index.php/news/article/plustechs_walking_ forest_m\%achine_03150832/.

[3] Y. Dai, E. Nakano, T. Takahashi, and H. Ookubo, "Motion control of leg-wheel robot for an unexplored rough terrain environment," in Proc. of 7th Int. Conf. on Advanced Robotics, vol. 2, 1995, pp. 911-916.

[4] H. Adachi, N. Koyachi, T. Arai, A. Shimizu, and Y. Nogami, "Mechanism and control of a leg-wheel hybrid mobile robot," in Proc. of Int. Conf. Intelligent Robots and Systems, 1999, pp. 1792-1797.

[5] S. J. Ylonen and A. J. Halme, "Workpartner - centaur like service robot," in Proc. of Int. Conf. Intelligent Robots and Systems, 2002, pp. 727-732.

[6] C. Grand, F. BenAmar, F. Plumet, and P. Bidaud, "Decoupled control of posture and trajectory of the hybrid wheel-legged robot hylos," in Proc. of Int. Conf. Robotics and Automation, 2004, pp. 5111-5116.

[7] G.Endo and S.Hirose, "Study on roller-walker (multi-mode steering control and self-contained locomotion)," in Proc. of Int. Conf. on Robotics and Automation, 2000, pp. 2808-2817.

[8] G. Endo and S. Hirose, "Study on roller-walker - adaptation of characteristics of the propulsion by a leg trajectory -," in Proc. of Int. Conf. on Intelligent Robots and Systems, 2008, pp. 1532-1537.

[9] M. Kumagai and K. Tamada, "Roller-walk of a biped robot using a truck with variable curvature,' in Proc. of ROBOMEC'07, 2007, pp. 2A1-J02(in Japanese).
[10] K. Hashimoto, Y. Sugahara, T. Hosobata, Y. Mikuriya, H. Sunazuka M. Kawase, H. Lim, and A. Takanishi, "Sliding motion of biped walking robots mounted on passive wheels - 1st report: Realization of swizzle motion by inline skates -," in Proc. of ROBOMEC'07, 2007, pp. 1A1-E11(in Japanese).

[11] G. Gabrielli and T. H. von Karman, "What price speed?" Mechanical Engineering, vol. 72, no. 10, pp. 775-781, 1950.

[12] K. Arikawa and S. Hirose, "Development of quadruped walking robot TITAN-VIII," in Proc. of Int. Conf. on Intelligent Robots and Systems, 1996, pp. 208-214.

[13] P. Gregorio, M. Ahmadi, and M. Buehler, "Design, control, and energetics of an electrically actuated legged robot," IEEE Transactions on Systems, Man, and Cybernetics - Part B: Cybernetics, vol. 27, no. 4, pp. 626-634, 1997.

[14] K. Dowling, "Limbless locomotion: Learning to crawl with a snake robot," Ph.D. dissertation, Robotics Institute, Carnegie Mellon University, Pittsburgh, PA, December 1997.

[15] D. Wettergreen, "Robotic walking on natural terrain: Gait planning and behavior-based control for statically-stable walking robots," Ph.D. dissertation, Robotics Institute, Carnegie Mellon University, Pittsburgh, PA, December 1995.

[16] T. McGeer, "Passive dynamic walking," International Journal of Robotics Research, vol. 9, no. 2, pp. 62-82, 1990.

[17] J. Yong, R. Smith, L. Hatano, and S. Hillmansen, "What price speed - revisited," INGENIA, vol. 22, pp. 46-51, 2005 (Available online).

[18] D. W. J.E. Bares, "Technical description, results, and lessons learned," Int. J. of Robotics Research, vol. 18, no. 7, pp. 621-649, 1999.

[19] S. Hirose and Y. Umetani, "The basic motion regulation system for a quadruped walking machine," in Design Engineering Tech. Conf. ASME, 1980, pp. 1-6.

[20] E. Krotkov and R. Simmons, "Performance of a six-legged planetary rover: Power, positioning, and autonomous walking," in Int. Conf. on Robotics and Automation, 1992, pp. 169-174.

[21] S. Talebi, I. Poulakakis, E. Papadopoulos, and M. Buehler, "Quadruped robot running with a bounding gait," in the 7th Int. Sym. on Experimental Robotics, 2000.

[22] S. Chernova and M. Veloso, "An evolutionary approach to gait learning for four-legged robots," in Int. Conf. on Intelligent Robots and Systems, 2004, pp. pp.2562-2567(velocity).

[23] M. S. Kim and W. Uther, "Automatic gait optimisation for quadruped robot," in the Austrasian Conference on Robotics and Automation, 2003, p. (duration).

[24] http:http://esupport.sony.com/US/perl/model-documents.pl?mdl= ERA201B1(battery).

[25] S. Collins, A. Ruina, R. Tedrake, and M. Wisse, "Efficient bipedal robots based on passive-dynamic walkers," Science, vol. 307, no. 5712, pp. 1082-1085, 2005.

[26] M. Wisse, "Three additions to passive dynamic walking; actuation, an upper body, and 3d stability," in Int. Conf. on Humanoid Robots, 2004, pp. 113-132.

[27] S. Collins and A. Ruina, "A bipedal walking robot with efcient and human-like gait," in Int. Conf. on Robotics and Automation, 2005, pp. 1995-2000.

[28] M. Raibert, H. B. Brown, M. Chepponis, J. Koechling, J. K. .Hodgins, D. Dustman, W. K. Brennan, D. S. B. adn C. M. Thompson, J. D. Hebert, W. Lee, and L. Borvansky, "Dynamically stable legged locomotion," in Technical Report 1179, LL-6, 1989, p. 134.

[29] J. A. Smith, I. Poulakakis, M. Trentini, and I. Sharf, "Bounding with active wheels and liftoff angle velocity adjustment," Int. J. of Robotics Research, vol. 29, no. 4, pp. 414-427, 2009.

[30] D. Campbell and M. Buehler, "Preliminary bounding experiments in a dynamic hexapod," Experimental Robotics VIII, pp. 612-621, 2003.

[31] D. R. Pugh, E. A. Ribble, V. J. Vohnout, T. E. Bihari, T. M. Walliser, M. R. Patterson, and K. J. Waldron, "Technical description of the adaptive suspension vehicle," Int. J. of Robotics Research, vol. 9, no. 2, pp. 24-42, 1990.

[32] K. J. Waldron and R. B. McGhee, "The adaptive suspension vehicle," IEEE Control Systems Magazine, vol. 6, no. 6, pp. 7-12, 1986.

[33] (2010) BostonDynamics - BigDog The Most Advanced Rough-Terrain Robot on Earth. [Online]. Available: http://www.bostondynamics.com/ robot_bigdog.html

[34] (2008) BigDog Overview. [Online]. Available: http://www. bostondynamics.com/img/BigDog_Overview.pdf 